\title{
Vulnérabilité des eaux souterraines à la pollution dans la ville de Dschang, Ouest-Cameroun
}

\author{
Franck Fabrice MBA ${ }^{1}$, Emile TEMGOUA ${ }^{1 *}$, Philippe Dupont KENGNE $^{2}$ et \\ Serge NATHEU KAMHOUA ${ }^{2}$ \\ ${ }^{I}$ Université de Dschang, Faculté d'Agronomie et des Sciences Agricoles, BP 222 Dschang, Cameroun. \\ ${ }^{2}$ Délégation Départementale du Ministère de l'Eau et de l'Energie de la Menoua, Cameroun. \\ ${ }^{*}$ Corresponding author; E-mail: etemgoual @yahoo.com
}

\section{RESUME}

Les populations de Dschang, ville moyenne de l'Ouest Cameroun, s'alimentent en eau principalement à travers les forages, les puits, les sources, les cours d'eau qui, malheureusement, ne présentent pas toujours l'eau de bonne qualité. Cette étude avait pour but d'évaluer la vulnérabilité des eaux souterraines à la pollution dans cette ville. La nature et l'origine des polluants ont été déterminées. Le sens de circulation des eaux souterraines a ensuite été déterminé avec la méthode DRASTIC, permettant ainsi d'évaluer le mode de transport et de propagation des polluants. Les résultats ont révélé que la circulation des eaux dans le sol est diffuse dans les zones à niveau topographique élevé et concentrique dans les zones à niveau topographique bas. Les eaux circulent dans la ville de Dschang selon les orientations NW - SE, N - S, NE - SW pour se concentrer à l'exutoire au SW, au niveau de l'escarpement de Foréké-Dschang. Les pressions anthropiques (ouvrage d'assainissement, agriculture urbaine) sont les principaux agents qui dénaturent la qualité des eaux. Les indices de vulnérabilités des eaux souterraines dans la ville de Dschang et ses environs varient de «faible » à «élevé » selon les conditions naturelles du milieu d'étude et la prise en compte de l'influence des activités anthropiques dans la zone. Ceci est confirmé par les teneurs des paramètres physico-chimiques et bactériologiques des eaux qui ne respectent pas toujours les normes. Cette étude permet de proscrire la réalisation des ouvrages hydrauliques sur les lignes de crêtes dans les zones topographiques élevées et le long des cours d'eaux suivant les points topographiques les plus bas dans les vallées drainées ou non.

(C) 2019 International Formulae Group. All rights reserved

Mots clés : Eau souterraine, activités anthropiques, pollution, vulnérabilité, Dschang, Cameroun.

\section{Vulnerability of groundwater to pollution in the city of Dschang, West Cameroon}

\section{ABSTRACT}

The populations of Dschang, a middle-class city in western Cameroon, collect water mainly from boreholes, wells, springs and waterways, which, unfortunately, do not always have good quality water. The purpose of this study was to assess the vulnerability of groundwater to pollution in the city of Dschang. The work consisted in first determining the direction of groundwater circulation via the DRASTIC method in 
order to evaluate the mode of transport and propagation of pollutants according to their nature and their origins. The results revealed that the circulation of water in the soil is diffuse in topslope and concentric in the downslope. The NW - SE, N - S, and NE - SW major flow directions intersect at SW, where they concentrate and then circulate to partially discharge at the Foréké - Dschang escarpment by emergence. The physicochemical and bacteriological quality of the city's waters is degraded by anthropic pressures (poor condition of the sanitation utilities, urban agriculture). The vulnerability of groundwater in and around Dschang varies from " low " to " high" in relation to anthropogenic pressures. Hence the installation of hydraulic infrastructures at the top of the high areas of the city is disadvantageous, because the circulation of groundwater is diffuse and likewise, it is preferential areas of protection and conservation to promote the recharge of groundwater. The location of these structures is also unfavorable in the lower zone because the circulation of the aquifers is concentric and close to the topographic surface.

(C) 2019 International Formulae Group. All rights reserved

Keywords: Groundwater, anthropogenic activities, pollution, vulnerability, Dschang, Cameroon.

\section{INTRODUCTION}

Depuis des temps immémoriaux, l'évaluation de l'eau a toujours été une préoccupation majeure. Aujourd'hui, la principale difficulté à laquelle le Cameroun est confronté n'est pas tant cet accès à l'eau mais plus précisément l'accès à l'eau potable. En effet, le problème se pose en terme de qualité des ressources en eau et c'est sur ce point que toutes les attentions sont tournées, au Cameroun comme ailleurs (DjeudaTchapnga et al., 2001). En effet, l'eau peut être le véhicule d'un très grand nombre d'agents pathogènes rejetés dans le milieu extérieur par les fèces humaines ou animales et peut ainsi être à l'origine de nombreuses maladies hydriques. L'OMS quantifie à 4 milliards le nombre d'épisodes de diarrhée survenus dans le monde et qui est responsable de 3,1 millions de décès, dont la grande majorité des enfants de moins de cinq ans (OMS, 2008). Une épidémie de choléra en janvier 2011 a affecté 2052 personnes dans huit des dix régions du Cameroun et a fait environ 85 décès (Pana express, 2011). En 2018, on déclare de nouveau environ 45 cas décelés à l'ExtrêmeNord du pays.

Dans le cas de la ville de Dschang, il existe une forte concentration de la population favorisée principalement par l'urbanisation et par la présence des structures scolaires et universitaires. La ville de Dschang compte environ $80 \quad 000$ habitants sur 220000 que renferme la Commune de Dschang (RGP, 2005). Cette dernière, comme toutes les autres agglomérations au Cameroun, n'est pas raccordée à un système d'égout ni d'assainissement commun, d'où la contamination probable des ressources en eaux du point de vue physico-chimique et bactériologique. Depuis 1990, des progrès sensibles ont été accomplis en matière d'accès à une source d'alimentation en eau potable. Selon WHO et UNICEF (2015), le taux de couverture en zone urbaine aurait évolué de $77 \%$ en 1990 à $92 \%$ en 2008 se traduisant par une augmentation de la population desservie de 3,8 millions à près de 10,0 millions. Mais en 2008, seulement $25 \%$ de la population urbaine était alimentée par branchement particulier, une proportion comparable à celle de 1990. En zone rurale, le taux de couverture serait passé de $31 \%$ à $51 \%$ entre 1990 et 2008. Pour pallier à ce manquement, les populations ont opté pour les méthodes alternatives d'approvisionnement (Temgoua et al., 2010). L'eau est puisée dans les forages, les puits, les sources et même les cours d'eau, laquelle eau n'est malheureusement pas toujours assurée en termes de qualité (Temgoua et al., 2009; Temgoua, 2011).

Il naît, dès lors, un problème lié à la quantité et à la qualité de l'eau disponible pour le développement socioéconomique durable de Dschang. La question liée à la pollution des ressources en eaux est couramment abordée par la méthode DRASTIC. Cette méthode a été développée aux États-Unis par Aller et al. (1987). Elle 
prend en compte plusieurs paramètres de l'environnement naturelle qui contrôlent les processus de contamination des aquifères (Chenini et al., 2015). L'insuffisance de la méthode réside au niveau de sa subjectivité des cotes attribuées à chaque paramètre et la variation de la classification selon les auteurs (Napolitano et Fabbri, 1996). Malgré la faiblesse de cette méthode, elle a fait ses preuves en Amérique (Aller et al., 1987), en Europe (Kaliraj et al., 2014), en Afrique (Akenji et al., 2015) et vu sa flexibilité adaptable à tous les environnements géologiques, elle a été testé récemment dans la localité de Foumbot (Fonka et al., 2019). Mais dans ce travail, les cotes attribuées demeurent dans une gamme fonction de la nature du milieu et la variation dans la classification tient compte de l'influence des activités anthropiques du milieu vu que le model de Aller et al est intrinsèque. Par cette méthode, des mesures pour remédier à la pollution et favoriser une gestion efficiente des ressources en eau pourraient être proposées.

\section{MATERIEL ET METHODES Site d'étude \\ Données géographiques}

La ville de Dschang est située dans la région de l'Ouest Cameroun (Figure 1). C'est le Chef-lieu du Département de la Menoua et de l'Arrondissement qui porte son nom. La zone d'étude constitue le noyau urbain de l'arrondissement de Dschang qui se situe entre les latitudes $5^{\circ} 25^{\prime}$ et $5^{\circ} 30^{\prime}$ Nord, entre les longitudes $10^{\circ} 00^{\prime}$ et $10^{\circ} 05^{\prime}$ Est et à une altitude moyenne de $1400 \mathrm{~m}$. Cette ville est située sur le versant Sud-ouest des Monts Bamboutos, et dominée par des bas plateaux fortement disséqués par de petites vallées parfois marécageuses (Kenfack et al., 2011).

Le climat est subéquatorial de type Equatorial Camerounien par altitude (froid et humide). La pluviométrie annuelle de la station de Dschang est d'environ $1830 \mathrm{~mm}$; il $y$ règne deux saisons: une saison pluvieuse allant de mars à octobre et une saison sèche comprenant les mois de janvier et février puis novembre et décembre. Le climat est généralement humide et fortement influencé par l'altitude. La température moyenne annuelle est de $21,9^{\circ} \mathrm{C}$. Les mois de février et mars sont les mois les plus chauds et les mois les plus frais sont juillet et août.

\section{Géologie et hydrogéologie}

La ville de Dschang est caractérisée par plusieurs activités volcaniques et des produits volcaniques de faciès variés (Kwekam, 2005): basaltes, trachytes, phonolites, rhyolite et les ignimbrites. Ses mêmes faciès affleurent dans la ville de Dschang en plus des formations du socle composées des granites et d'orthogneiss; d'où l'appellation de complexe granitogneissique.

Les formations sédimentaires se trouvent dans la localité de Ngwa (Kenfack et al., 2011). Elles sont intercalées entre deux épisodes volcaniques et représentée par des dépôts détritiques de conglomérats, de brèches, d'argiles ligniteuses, des gleys pélitiques et des ardoises carbonatées, enfin une couche de conglomérats et des gleys arkosiques.

Principalement deux types de nappes existent dans la zone d'étude (Ngako, 2002) : les nappes d'altération qui se retrouvent à la base des profils de sol et dans lesquelles se font les puits d'eau; les nappes de fractures qui se localisent dans les discontinuités lithologiques et qui résultent des déformations cassantes qu'a subi la région (pour les roches du socle) ou des fissures qui délimitent les prismes (pour le cas des roches volcaniques). Les structures géologiques ont une grande influence sur la dynamique et la qualité des eaux dans cette région.

\section{Modes d'approvisionnement en eau potable dans la ville de Dschang}

Le développement accéléré de la ville de Dschang au cours de ces dernières années a été marqué par un décalage entre l'offre et la demande en matière de services urbains. Le taux de couverture en matière d'accès à l'eau est inférieur à $50 \%$ (PIGEDEA, 2017). Cette situation conduit 
les populations à assurer leur approvisionnement en eau (AEP) par leurs propres moyens. La multiplication des fosses à aisance (latrines et fosses sceptiques non fonctionnelles pour la plupart) et leur manque d'entretien, notamment non loin des points d'eau (sources, forages, puits) présentent un risque sanitaire permanent. Il y a sur le territoire de la commune de Dschang 31 Mini-réseaux alimentant 68 bornes fontaines et 41 forages. La plupart de bornes fontaines et de forages sont non fonctionnels. Les puits ordinaires sont presque dans toutes les concessions de la commune, en particulier dans le centre urbain. Ils sont de trois types à savoir: les puits non aménagés (sans margelle ni poulie), les puits semi aménagés (avec margelle sans couvercle et sans poulie) et les puits aménagés (margelle avec couvercle et poulie présente) servant à fournir l'eau pour les travaux domestiques (vaisselle, lessive, cuisine, douche).

\section{Méthodes}

Ce travail s'est appuyé fortement sur les caractéristiques d'un grand nombre de coupes de forage collectées dans la base de données de la Délégation départementale de l'eau et de l'énergie pour la Menoua. Par la suite, les descentes sur le terrain ont permis la description des profils pédologiques, des mesures piézométriques, les mesures des paramètres in situ et la collecte des échantillons de sol et d'eau. Les paramètres de pollution (polluants agricoles, polluants bactériologiques) dans les eaux récoltées ont été analysés au Laboratoire d'Analyse des Sols et de Chimie de l'Environnement (LABASCE) de l'Université de Dschang. Les différentes côtes ont permis d'établir à l'aide du logiciel Sufer 13 (C) la carte des lignes d'équipotentielle et des lignes de courant afin de présenter les niveaux et les sens d'écoulements généraux des eaux souterraines. Les modes de transport et de propagation des différents polluants ont été déterminés par la méthode DRASTIC. Les grandeurs physiques du sol suivantes qui conditionnent l'écoulement de la nappe ont été utilisées: densité apparente (D), teneur en eau volumique du sol $(\theta)$ et porosité $(\mathrm{P})$. Les paramètres de la nappe qui jouent un rôle primordial dans la circulation des eaux souterraines et des polluants ont été calculés : Rabattement résiduel ( $\mathrm{Sr}$ ), débit spécifique $(\mathrm{Q} / \mathrm{s})$, transmissivité $(\mathrm{T})$ et perméabilité (K).

Rabattement, $\mathrm{S}_{\mathrm{r}}=(0,183 \mathrm{Q} / \mathrm{T}) \log \left(1+\mathrm{t}_{\mathrm{p}} / \mathrm{t}_{\mathrm{r}}\right)$ (1)

Débit spécifique $(Q / s)$ et Transmissivité (T), selon Hamm et al. (2005).

$\mathrm{Q}_{/ \mathrm{s}}=\mathrm{Q} / \mathrm{S}$

La relation entre $T$ et $Q_{/ s}$ est donnée à l'équation (3) suivante selon Thomasson et al. (1960) in Rot-Zoll et El-Kadi (2008):

$T=A_{I} \cdot Q_{/ s}$

Où la constante $A_{1}$ sans dimension est comprise entre 0,9 et 1,52 , avec une moyenne de 1,18 .

La transmissivité de la nappe $(\mathrm{T})$ est liée à la perméabilité $(\mathrm{K})$ par la relation: $\quad \mathrm{T}=\mathrm{KD}$ soit $\mathrm{K}=\mathrm{T} / \mathrm{D}$

Avec $\mathrm{D}=$ épaisseur de la nappe.

\section{Evaluation de la vulnérabilité des nappes par la méthode DRASTIC modifiée}

La méthode DRASTIC évalue la sensibilité de l'eau souterraine à être affectée par une contamination provenant directement de la surface. Pour évaluer la vulnérabilité des eaux souterraines suivant la méthode DRASTIC, les données suivantes ont été nécessaires: niveaux de la nappe, stratigraphie de la zone vadose et de l'aquifère, recharge, données pédologiques, topographie et conductivité hydraulique de l'aquifère (Tableau 1).

\section{Aires de protections concernées}

Les aires de protections concernées sont : Immédiates, intermédiaires et éloignées. L'acronyme anglais DRASTIC correspond aux sept paramètres physiques et hydrogéologiques considérés dans le calcul des indices de vulnérabilité. Une valeur numérique appelée poids comprise entre 1 et 5 reflète le degré d'influence de chacun de ces paramètres dans le calcul des indices. Ainsi, la profondeur de la nappe et la nature 
de la zone vadose exercent le plus d'influence, suivie de la recharge. Par contre, la topographie est le paramètre ayant le moins d'impact, suivi du type de sol. Enfin, la nature du milieu aquifère et sa conductivité ont pour leur part un poids modéré.

Une cote variant de 1 à 10 , définie en fonction d'intervalles de valeurs, est associée à chacun des paramètres. Elles sont compilées dans le tableau des cotes des paramètres (Tableau 1) pour le calcul des indices DRASTIC. Plus la cote est élevée, plus la vulnérabilité est élevée.

Le calcul de l'indice de vulnérabilité par la méthode DRASTIC correspond ainsi à la somme des cotes attribuées aux différents paramètres multipliées par leur poids. Les valeurs de l'indice peuvent varier entre 23 et 226. Comme pour les cotes, plus l'indice est élevé, plus la vulnérabilité intrinsèque de l'eau souterraine est élevée.

\section{Equation}

Indice DRASTIC: DcDp + RcRp + AcAp + $\mathrm{ScSp}+\mathrm{TcTp}+\mathrm{IcIp}+\mathrm{CcCp}$

Où l'indice $c$ représente la cote et l'indice $p$ représente le poids.

\section{Détermination des cotes}

La profondeur de l'aquifère est un facteur important dans l'étude de la vulnérabilité, car toute atténuation du polluant s'opère en relation avec la profondeur. La profondeur de la nappe phréatique (D) a été mesurée dans 105 ouvrages hydrauliques (97 puits et 8 forages) sélectionnés dans la zone d'étude au cours des mois d'Avril et Mai de l'année 2018, et ceci grâce à l'utilisation d'une sonde lumineuse dans le cas des puits. Pour les aquifères en conditions de nappe semicaptive, comme l'infiltration verticale est tout de même significative à l'intérieur de la couche semi-perméable juxtaposant les aquifères, ils ont été considérés en conditions de nappe libre pour la détermination de la cote. Pour le cas des aquifères de roches fracturées en conditions de nappe captive, la profondeur de la base de l'aquitard immédiatement au-dessus a été considérée. La profondeur des nappes profondes (D) est obtenue des relevés de 8 forages. Les mesures ont été faites en mètres. Les positions ont été relevées à l'aide d'un GPS.

Comme la profondeur de la nappe libre subit des variations saisonnières, par principe de précaution, l'utilisation des valeurs les plus faibles pour le calcul des indices est effectuée. Ces valeurs se rapprochent des données collectées sur le terrain car la période où les nappes sont en recharge par rapport aux saisons.

\section{Estimation de la recharge}

Le transport des polluants de la zone non saturée jusqu'à l'aquifère est produit par la dissolution pendant la recharge en eau. La quantité d'eau qui arrive au niveau de l'aquifère constitue la recharge nette, facteur important de la vulnérabilité. Elle se mesure en $\mathrm{mm} / \mathrm{an}$. Cette recharge représente le mécanisme primaire du transport des contaminants dans la nappe et dépend étroitement du type de sol en présence. La source primaire de recharge des nappes de la zone d'étude est la précipitation. L'estimation de la recharge annuelle (en $\mathrm{mm} / \mathrm{an}$ ) a été faite en utilisant les équations de Williams et de Kissel (Jha et Sebastain, 2005).

Equation: $\mathrm{PI}=(\mathrm{P}-15,05)^{2} /(\mathrm{P}+22,57)$

La formule (6) concerne les sols argileux secs, limono- sableux et sablo-limoneux où PI est l'index de percolation et $\mathbf{P}$ la précipitation totale annuelle.

La recharge nette des aquifères est estimée par la formule du bilan hydrique.

Equation: $\mathrm{P}=\mathrm{ETP}+\mathrm{R}+\mathrm{I}$

Avec :

$\mathrm{P}$ : précipitation annuelle en $\mathrm{mm}$

$\mathrm{R}$ : ruissellement en $\mathrm{mm}$

$\mathrm{I}=1 / 3 \mathrm{R}$

L'évapotranspiration annuelle est déterminée par la formule annuelle de Turc (1961).

L'estimation de la recharge régionale, ou à l'échelle de l'aquifère, a été considérée aux fins $\mathrm{du}$ calcul de l'indice DRASTIC. 
F. F. MBA et al. / Int. J. Biol. Chem. Sci. 13(5): 39-56, 2019

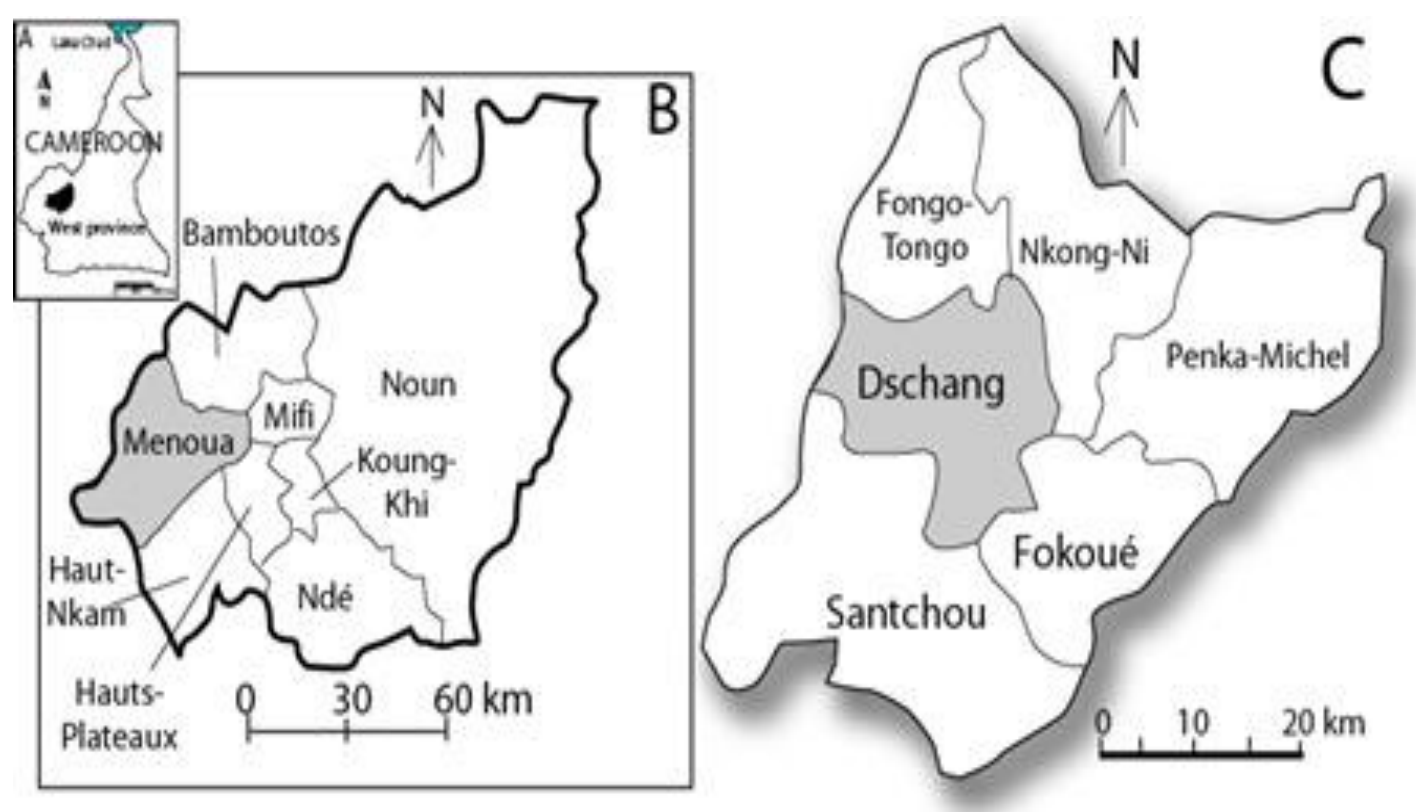

Figure 1: Localisation de la ville de Dschang au Cameroun (A), dans la Région de l’Ouest (B) et dans le Département de la Menoua (C). 
Tableau 1 : Cotes des paramètres pour le calcul de l'indice DRASTIC.

\begin{tabular}{|c|c|c|c|c|c|c|c|}
\hline COTES & $\mathbf{D}$ & $\mathbf{R}$ & $\mathbf{A}$ & $\mathbf{S}$ & $\mathbf{T}$ & I & C \\
\hline & $\begin{array}{l}\text { Profondeur } \\
\text { de la nappe } \\
(\mathrm{m})^{(1)}\end{array}$ & $\begin{array}{l}\text { Recharge } \\
\text { (mm/an) }\end{array}$ & $\begin{array}{l}\text { Nature du milieu aquifère } \\
(\text { Intervalle de côte })^{(2)}\end{array}$ & Type de sol $^{(3)}$ & $\begin{array}{l}\text { Pente du } \\
\text { terrain }(\%)\end{array}$ & $\begin{array}{l}\text { Nature de la zone vadose } \\
\text { (intervalle de côte) }^{(2)}\end{array}$ & $\begin{array}{l}\text { Conductivité } \\
\text { hydraulique de } \\
\text { l'aquifère (m/jr) }\end{array}$ \\
\hline 1 & 31 et plus & De 0 à 50 & & Argile & 18 et plus & - Couche confinante (1) & De 0,04 à 4 \\
\hline 2 & De 23 à 31 & & - Schiste massif (1-3) & Terre noire & & & De 4 à 12 \\
\hline 3 & De 15 à 23 & De 50 à 100 & $\begin{array}{l}\text { - Roches ignées ou } \\
\text { métamorphiques }(2-5)\end{array}$ & Loam argileux & De 12 à 18 & $\begin{array}{l}\text { - Silt ou argile (2-6) } \\
\text { - Schiste }(2-5)\end{array}$ & \\
\hline 4 & & & $\begin{array}{l}\text { - Roches ignées ou } \\
\text { métamorphiques altérées (3-5) }\end{array}$ & Loam silteux & & $\begin{array}{l}\text { - Roches ignées ou } \\
\text { métamorphiques (2-8) }\end{array}$ & De 12 à 29 \\
\hline 5 & De 9 à 15 & & $\cdot$ - Till (4-6) & Loam & De 6 à 12 & & \\
\hline 6 & & De 100 à 180 & $\begin{array}{l}\text { - Lits de grès, de calcaire et de } \\
\text { schiste }(5-9) \\
\text { - Grès massif (4-9) } \\
\text { - Calcaire massif (4-9) }\end{array}$ & Loam sableux & & $\begin{array}{l}\text { - Calcaire }(2-7) \\
\text { - Grès }(4-8) \\
\text { - Lits de calcaire, de grès et de } \\
\text { schiste }(4-8) \\
\text { - Sable et gravier avec silt et } \\
\text { argile }(4-8)\end{array}$ & De 29 à 41 \\
\hline 7 & De 4,5 à 9 & & & Argile fissurée & & & \\
\hline 8 & & De 180 à 250 & - Sable et gravier (4-9) & Tourbe & & - Sable et gravier (6-9) & De 41 à 82 \\
\hline 9 & De 1,5 à 4,5 & 250 et plus & - Basalte (2-10) & Sable & De 2 à 6 & - Basalte (2-10) & \\
\hline 10 & De 0 à 1,5 & & - calcaire karstique (9-10) & $\begin{array}{l}\text { Sol mince ou roc } \\
\text { ou gravier }\end{array}$ & De 0 à 2 & - Calcaire karstique (8-10) & 82 et plus \\
\hline Poids & 5 & 4 & 3 & 2 & 1 & 5 & 3 \\
\hline
\end{tabular}

Source : Aller et al. (1987). Légende des marques sur les titres des colonnes du tableau 1 :

(1) En conditions de nappe captive, la profondeur de la nappe correspond au toit de l'aquifère.

(2) Chaque matériau est classé selon la cote type proposée par la méthode DRASTIC. Elle propose aussi un intervalle de cote, indiqué entre parenthèse dans ce tableau.

(3) Environ le premier mètre de dépôt à partir de la surface du sol.

(4) Portion souterraine entre le sol et le niveau de la nappe ou entre le sol et le toit de l'aquifère dans le cas d'une nappe captive. 


\section{RESULTATS}

Nature et origine des polluants dans les eaux de la ville de Dschang

La construction de nouvelles habitations se fait de façon désordonnée, surtout dans les zones fragiles. La zone à habitat spontané est constituée des quartiers Mingmeto, Ngui, Haoussa, New-town Foto et une partie du quartier Mingou. Le marché B dans la zone marécageuse est presqu'une poubelle. Dans tous ces cas, les rivières, à plusieurs endroits de la ville, sont le lieu de vidange de WC; certaines buvettes focalisent leurs toilettes en direction des cours d'eau ; la structure pénitentiaire le fait également. Le rapport de l'Agence Municipale de Gestion des Déchets (AMGED, 2017) indique un taux de collecte de $30 \%$ pour un total de 60 tonnes d'ordures produits par jour, soit $70 \%$ déversés dans les cours d'eau et les espaces verts de la ville. L'agriculture urbaine se fait dans tous les marécages, avec un fort input en engrais chimique et fiente de poule. De façon globale, les polluants du secteur d'étude sont de plusieurs natures (polluants biologiques, polluants chimiques, polluants organiques et polluants persistants) et ont plusieurs origines.

\section{Détermination de la vulnérabilité des nappes de la ville de Dschang}

Le Tableau 2 récapitule l'ensemble des paramètres DRASTIC obtenus. Les aquifères de la zone d'étude sont composites et contiennent assez de sable, sont de ce fait perméables et assez d'argile pour retenir les polluants.

Le calcul de l'indice DRASTIC suivant le Tableau 2 dans les zones basses (1300 à $1354 \mathrm{~m})$, moyenne (1354 à $1400 \mathrm{~m})$ et haute $(1400$ à 1600) prend en considération les formations plutoniques et les formations volcaniques dont les logs lithologiques sont représentés à la Figure 2.

$$
\text { L'analyse des lignes }
$$

d'équipotentielles et des lignes de courants telles que présentées à la Figure 3 montre que les polluants peuvent se propager sur de grandes distances via l'étendue des systèmes aquifères d'altérité qui sont en majorité limités par les cours d'eau drainant en plus $\mathrm{du}$ fait que ces cours d'eau sont soutenues par ces nappes en période d'étiage. Mais un cas particulier se dégage dans la zone du lac municipal de Dschang. Le lac est l'exutoire de son bassin versant et est en communication avec la nappe superficielle. Ceci est justifié par le fait que la quasitotalité des puits situés dans la zone basse du bassin versant et proche du lac, ont des cotes piézométriques sensiblement similaires à la cote obtenue sur les berges du lac par soustraction de l'altitude du niveau d'eau à la valeur de la profondeur moyenne du lac suivant les transepts. Il est donc probable que le lac, entant que régulateur des eaux de ruissellement dans son environnement, pollue la nappe par contact mais aussi soutient la nappe et vice versa.

La Figure 3 et les observations de terrain montrent que les eaux de cette zone se déversent en aval via une chute située après le centre pénitencier de Dschang. Ce qui permet d'émettre l'hypothèse selon laquelle la zone du lac repose sur une structure résistante. Partant du log lithologique de cette zone, la structure de terrain pourrait être désavantageuse pour la production d'eau potable en quantité et en qualité suffisante via les forages.

En définitive, la vulnérabilité ne dépend que des seules conditions physiographiques (étude des formes de relief) et hydrogéologiques du milieu mais peut être adaptée au contexte de l'étude. Les polluants sont essentiellement transportés en direction des bas-fonds par l'eau de ruissellement pour ce qui est des polluants biologiques, chimiques, physiques et persistants. Seuls les polluants en phase dissouts sont transportés par percolation à travers les terrains qui surmontent la zone saturée. La vitesse de propagation et de destruction des polluants dépend de la nature de l'aquifère, de la pénétration d'eau de rivière polluée ou contact d'eau de rivière polluée avec la nappe et des inondations qui introduisent directement les polluants dans l'aquifère via le puits ou nappe qui affleure. 
Un aquifère peut être vulnérable mais à l'absence de pressions industrielles, agricoles et domestiques, ne peut être atteint par une pollution.

\section{Ecoulement général des eaux souterraines dans la ville de Dschang}

La Figure 3 illustre le sens général de circulation des eaux de la nappe dans la ville de Dschang et ses environs. Les activités de terrain sur cette partie montrent que les nappes d'altérites sont discontinues mais suivent le sens général d'écoulement des eaux car elles sont limitées les unes des autres par les cours d'eau à la surface topographique. Les nappes profondes sont par contre plus vastes, plus profondes et respectent également le sens de circulation des eaux. Toutes ces deux nappes pendent en direction du Sud-ouest suivant la direction du cours d'eau Menouet.

$\mathrm{Au}$ total, les eaux souterraines de la zone d'étude s'écoulent de manière diffuse dans les zones de haut potentiel telles que les zones à surfaces topographiques élevées puis de manière concentrique dans les zones de faible potentiel. Les sens majeurs de circulation sont orientés NW - SE, N - S, NE - SW pour se concentrer au SW afin de circuler vers la plaine des Mbô. La décharge est effectuée en partie au niveau de l'escarpement de Foréké - Dschang (Figure 3). Les différentes nappes présentent des indices de vulnérabilité allant de faible à élevé (Figure 4).

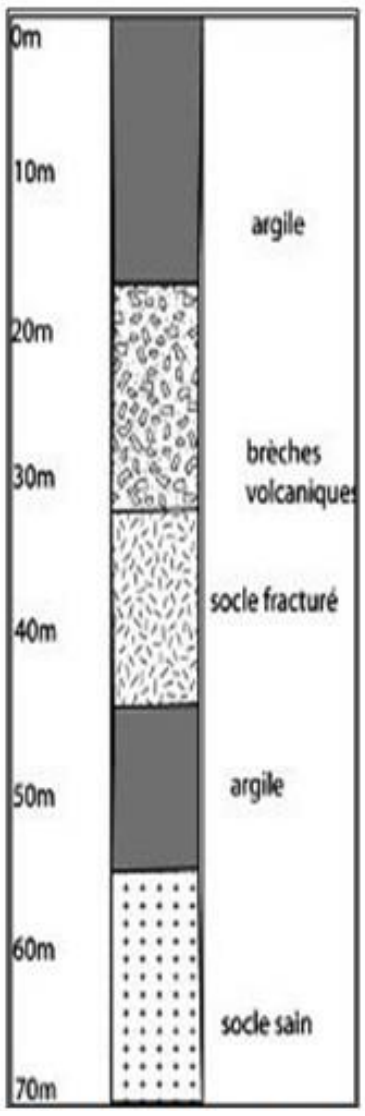

Zone basse

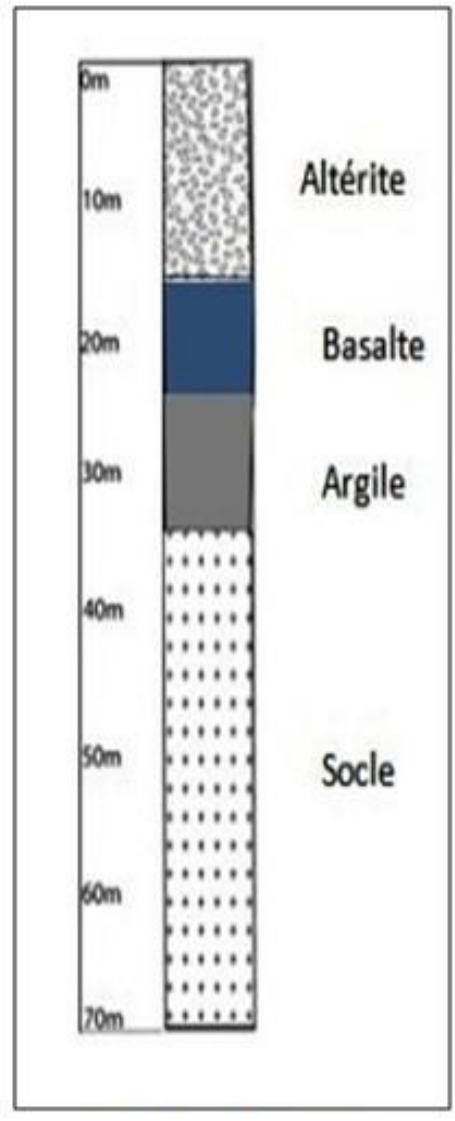

Zone moyenne

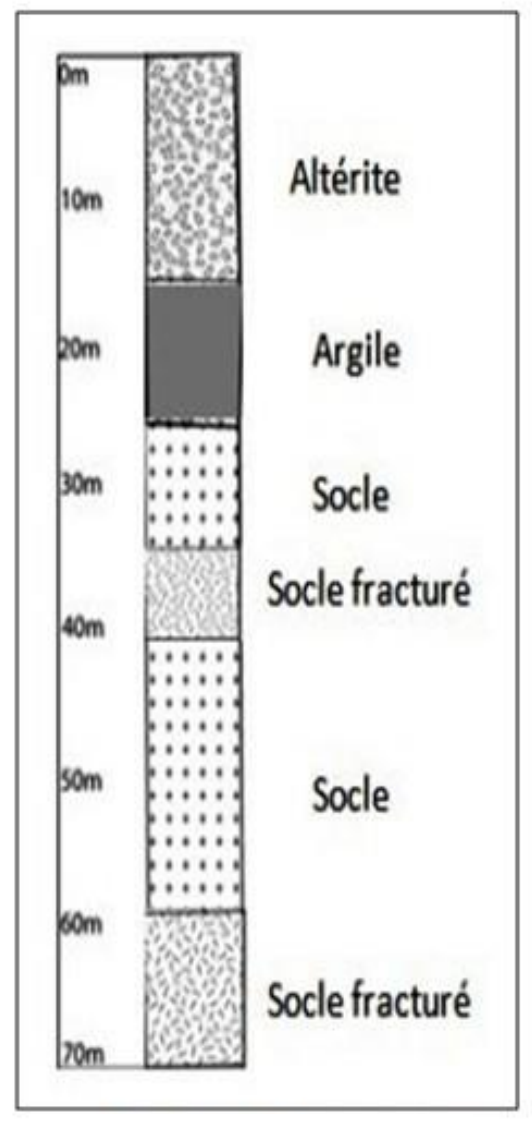

Zone haute

Figure 2: Logs lithologiques des zones de propriétés hydroliques similaires de la zone d'étude. 
Tableau 2: Récapitulatif des différents paramètres de l'indice DRASTIC.

\begin{tabular}{|c|c|c|c|c|c|c|c|c|c|c|c|c|c|c|}
\hline & \multicolumn{2}{|l|}{ D } & \multicolumn{2}{|l|}{$\mathbf{R}$} & \multicolumn{2}{|c|}{$\mathbf{A}$} & \multicolumn{2}{|l|}{$\mathbf{S}$} & \multicolumn{2}{|l|}{$\mathbf{T}$} & \multicolumn{2}{|l|}{$\mathbf{I}$} & \multicolumn{2}{|l|}{$\mathrm{C}$} \\
\hline & $\begin{array}{l}\text { Classe D } \\
\text { en }(m)\end{array}$ & $\begin{array}{l}\text { Note } \\
\text { (D) }\end{array}$ & $\begin{array}{l}\text { Classe } \\
\text { R en } \\
(\mathbf{m m} / \mathbf{a n})\end{array}$ & $\begin{array}{l}\text { Note } \\
\text { (R) }\end{array}$ & Classe A & $\begin{array}{l}\text { Note (A) } \\
\text { sur } \\
\text { Rv/Rp }\end{array}$ & Classe $\mathbf{S}$ & $\begin{array}{l}\text { Note } \\
\text { (S) }\end{array}$ & $\begin{array}{l}\text { Plage de } \\
\text { pente } \mathrm{T} \text { en } \%\end{array}$ & $\begin{array}{l}\text { Note } \\
(\mathbf{T})\end{array}$ & $\begin{array}{l}\text { Nature } \\
\text { lithologique } \\
\text { (I) }\end{array}$ & $\begin{array}{l}\text { Note } \\
\text { (I) }\end{array}$ & $\begin{array}{l}\text { Conductivité } \\
\text { hydraulique } \\
\text { (C) en m/jr }\end{array}$ & $\begin{array}{l}\text { Note } \\
\text { (C) }\end{array}$ \\
\hline & $0-1,5$ & 10 & $0-50$ & 1 & Latérite & 9 & Argileuse & 1 & 0 à 2 & 10 & $\begin{array}{l}\text { Concrétion } \\
\text { ferrugineuse }\end{array}$ & 3 & 0,1614 & 1 \\
\hline & $1,5-4,5$ & 9 & $50-100$ & 3 & Altérité & $8 / 7$ & $\begin{array}{l}\text { Argilo- } \\
\text { limoneuse }\end{array}$ & 2 & 2 à 6 & 9 & $\begin{array}{l}\text { Argile } \\
\text { sableuse }\end{array}$ & 4 & 0,0447 & 1 \\
\hline & $4,5-9$ & 7 & $\begin{array}{l}100- \\
180\end{array}$ & 6 & Allotérite & $6 / 5$ & $\begin{array}{l}\text { Limono- } \\
\text { argileuse }\end{array}$ & 3 & 6 à 12 & 7 & $\begin{array}{l}\text { Argile et } \\
\text { limon avec } \\
\text { mélange de } \\
\text { sable et } \\
\text { gravillon }\end{array}$ & 5 & 0,7079 & 1 \\
\hline & $9,0-15,0$ & 5 & $\begin{array}{l}180- \\
250\end{array}$ & 8 & Isaltérite & 4 & Limoneuse & 4 & 12 à 18 & 4 & Ignimbrite & 7 & 0,9637 & 1 \\
\hline & $15-23$ & 3 & $>250$ & 9 & $\begin{array}{l}\text { Socle } \\
\text { gneissique }\end{array}$ & 3 & $\begin{array}{l}\text { Limono- } \\
\text { argilo- } \\
\text { sableuse }\end{array}$ & 6 & $>18$ & 2 & $\begin{array}{l}\text { Altérite } \\
\text { latéritique }\end{array}$ & 8 & 0,0656 & 1 \\
\hline & $23-30$ & 2 & & & & & $\begin{array}{l}\text { Argilo- } \\
\text { sableuse }\end{array}$ & 7 & & & Basalte & 9 & 0,0754 & 1 \\
\hline & $>30$ & 1 & & & & & Sablo-argileux & 9 & & & & & & \\
\hline Poids & & 5 & & 4 & & 3 & & 2 & & 1 & & 5 & & 3 \\
\hline
\end{tabular}

$\operatorname{Rv}$ (nature de l'aquifère sur roche volcanique)

$\mathrm{Rp}$ (nature de l'aquifère sur roche plutonique) 
F. F. MBA et al. / Int. J. Biol. Chem. Sci. 13(5): 39-56, 2019

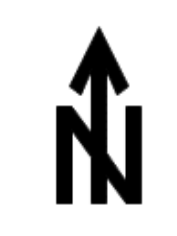

Légende

nappe perchée

ligne de courant

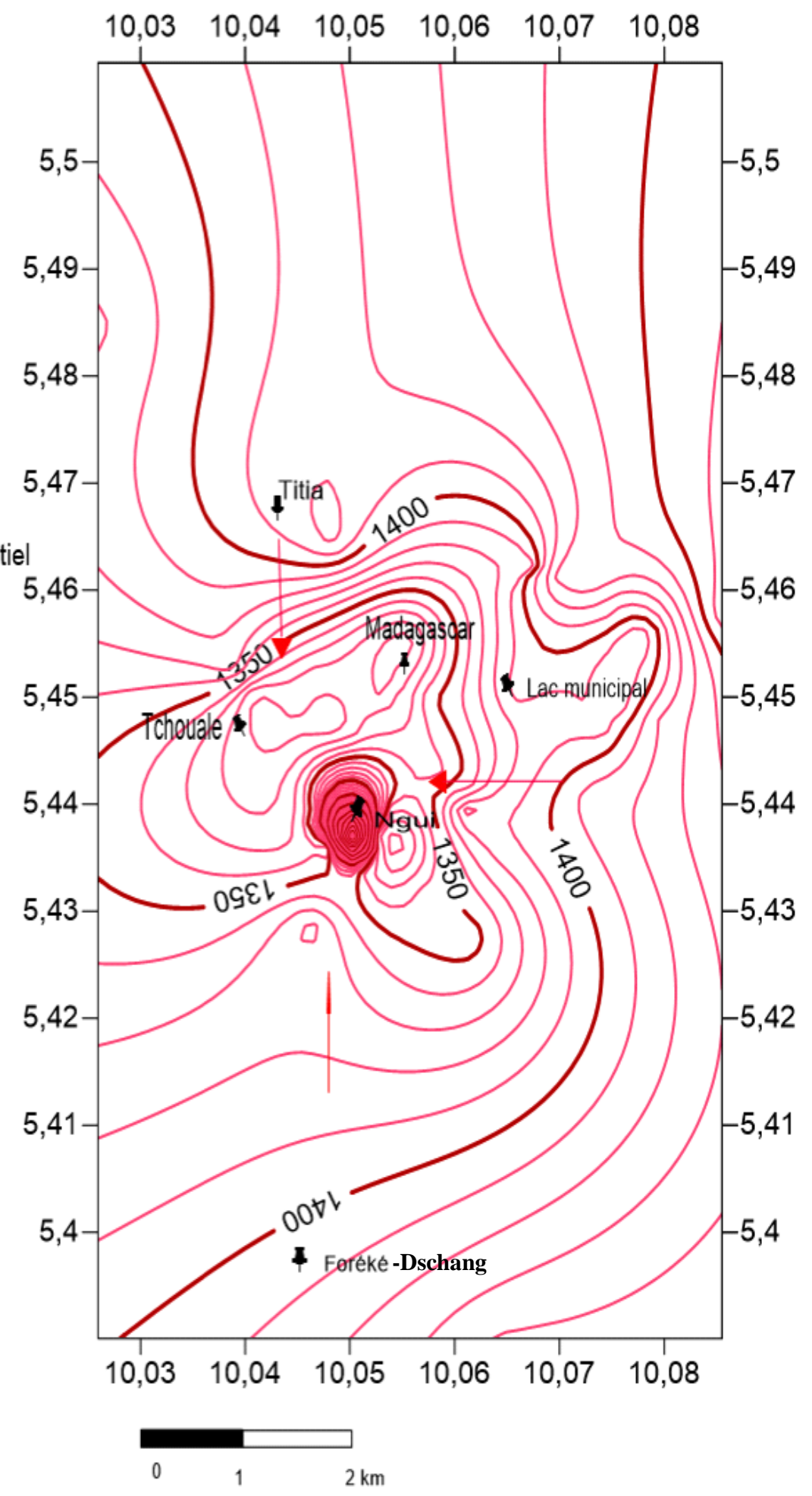

Figure 3: Carte piézométrique de la ville de Dschang. 


\section{DISCUSSION}

Les eaux souterraines de la zone d'étude s'écoulent de manière diffuse dans les zones de haut potentiel tel que les zones à surfaces topographiques élevées contribuant ainsi à la recharge des nappes conformément aux travaux de Loukman et al. (2017) puis de manière concentrique dans les zones de faible potentiel (Figure 3). Les résultats obtenus montrent que l'indice de vulnérabilité dans la majeure partie des aquifères d'altérites est supérieur à 100 (Tableau 4). Le degré de vulnérabilité d'après Ewodo et al. (2016) et conforme à celui initié par Aller et al. (1987) varie de faible sur les versant jusqu'au sommet à moyen dans les zones basses de convergence des eaux. Ces résultats s'éloignent de la réalité du terrain (Figure 4) mais reflètent plutôt un environnement avec absence des pressions anthropiques puisque le calcul à la fin de l'indice DRASTIC concerne la vulnérabilité intrinsèque qui est invariable au cours du temps. L'indice de calcul ne tient compte que des caractères propres au milieu naturel. Ce premier cas reflète la vulnérabilité intrinsèque des aquifères de la ville de Dschang et ses environs conformément aux travaux de Aké et al. (2009).

Par contre, le degré de vulnérabilité d'après Hamza et al. (2008) se rapproche de la réalité du terrain (Figure 4). En effet, les valeurs d'indice élevé et de degré de vulnérabilité également élevé de la zone basse selon la nature du terrain se justifient par les résultats d'analyse bactériologique effectués dans ces zones (Tableau 3). Le degré de vulnérabilité est moyen sur granitogneiss et élevé sur basalte dans la zone moyenne or cela est dû au fait que les puits prélevés avaient des profondeurs variant de 5 à $9 \mathrm{~m}$. La zone de la chefferie
Foréké-Dschang était plus représentée pour ce qui est des aquifères sur granitogneiss. La zone du lac municipal prolongé jusqu'à Menouet puis jusqu'à Keleng I et II dans la zone moyenne présente également des bandes de sols hydromorphes le long des affluents du lac. La zone haute sur granitogneiss est faible, différent de celle sur basalte qui est moyenne. L'illustration dans ce cas pourrait partir du fait que la nature lithologique des formations superficielles sur granitogneiss est essentiellement argileuse. La morphologie du relief présente des collines en demi-orange, impliquant le ruissellement privilégié à l'infiltration. Pourtant, les formations sur basalte sont plutôt constituées d'épaisses couches latéritiques allant jusqu'à $20-25 \mathrm{~m}$ d'épaisseur. Ces altérites latéritiques sont formées de macro pores qui permettent une bonne perméabilité mais une mauvaise filtration. La nappe profonde est plus vaste et présente l'indice faible.

Les terrains de la zone d'étude sont composés d'argiles, de sables et de limons comme composantes majeures. La pollution s'y propage donc de façon très variable. La perméabilité de l'ensemble est faible. La pollution se propage lentement dans les aquifères des terrains métamorphiques constitués de gneiss. Les zones sur site d'étude où la topographie donne lieu aux collines, la surface piézométrique de la nappe prend la forme d'un «dôme» et favorise l'écoulement divergent tel qu'illustré à la Figure 3. Ces zones sont les parties ayant les cotes piézométriques les plus élevées de la surface piézométrique et sont alimentées principalement par l'infiltration des eaux de pluie sans toutefois être influencées par les cours d'eau de la surface topographique. La pollution des eaux est dont non seulement influencée par 
la nature du terrain et des différents processus hydrochimiques entre les éléments présents dans les aquifères tels que démontré par Njueya et al. (2012) à l'issu de leurs travaux dans le bassin sédimentaire de Douala mais aussi par l'impact anthropique provenant de la surface tel que soutenu par Moussa et al. (2018) à l'issu de leurs travaux portant sur la caractérisation physicochimique des eaux des aquifères à Zinder au Niger. Le constat permet d'affirmer aisément que l'indice DRASTIC dans la dernière colonne du tableau d'après Hamza et al. (2008) reflète la vulnérabilité spécifique aux actions anthropiques tel que démontré par Bézèlgues et al. (2002), et Allier et al. (2008).

\section{Mesures palliatives à la pollution des eaux dans la ville de Dschang}

La majeure partie de la pollution des nappes dans la ville de Dschang provient de la pollution de surface. Les engrais utilisés tout le long de ce versant des monts Bamboutos sont lessivés en direction de la zone d'étude. La contamination bactériologique des eaux provient des fèces par contact des fosses d'aisance avec la nappe. La solution consiste donc à limiter l'usage des engrais en amont en apportant les doses suffisantes au sol. L'amendement des sols doit se faire immédiatement avant le retournement du sol et/ou le sarclage afin de limiter le lessivage des intrants agricoles. Les mesures précédentes permettent d'identifier les sources de contamination et de les éliminer tel que proposé par Temgoua (2011). Les fosses d'aisance des zones où la nappe est proche de la surface topographique doivent être supprimées au profit des toilettes réinventées (à vidange sur site) ou latrines à parois et fonds imperméables. Mais la solution la plus facile et moins coûteuse reste et demeure la prévention de la pollution des eaux souterraines.

L'une des dispositions consiste à préserver les zones de recharge et de décharge de la nappe. Cette préservation passe par la protection des lignes de crêtes contre les activités anthropiques ainsi que les bas-fonds. Les zones basses concentrent les polluants drainés; il convient d'éviter la fertilisation chimique en agriculture dans les bas-fonds au centre ou en périphérie urbaine. Les puits sont également les points de contacts de l'atmosphère avec la nappe et contribue à la pollution des eaux souterraines par de mauvaises pratiques des règles d'hygiène et le cumul des habitats. La réalisation des puits aménagés est dès lors importante pour limiter la pollution des eaux souterraines.

Les opérations de réalisation des fosses d'aisance dans la ville doit être éloigné des toilettes au moins de $15 \mathrm{~m}$ de part et d'autre avec une base située à $7 \mathrm{~m}$ audessus de la zone saturée de l'aquifère. Pour les zones où la nappe affleure, éviter le contact des latrines à fond perdu avec la nappe. Pour cela, prôner la réalisation de toilettes portatives ou bien équiper le fond de la fosse d'au moins $90 \mathrm{~cm}$ de sable fin tel que prescrit par l'OMS (Baba-Moussa, 1994). 
Tableau 3: Paramètres bactériologiques des eaux de forages de la ville de Dschang.

\begin{tabular}{|c|c|c|c|c|c|c|}
\hline Localité & Balevouni & Lingang & Tchouale & $\begin{array}{l}\text { Foréké- } \\
\text { Dschang }\end{array}$ & Nzen-keussok & 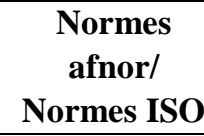 \\
\hline Dénomination & résultats & résultats & résultats & résultats & résultats & \\
\hline $\begin{array}{l}\text { Escherichia } \\
\text { coli/100ml }\end{array}$ & Absence & Absence & $\begin{array}{l}\text { Présence d'E. } \\
\text { coli }\end{array}$ & Absence & Absence & Absence \\
\hline $\begin{array}{l}\text { Staphylocoque/ } \\
\text { 100ml }\end{array}$ & Absence & Absence & Absence & Absence & Absence & $<20 \mathrm{ufc} / \mathrm{ml}$ \\
\hline $\begin{array}{l}\text { Salmonellaes/1 } \\
\text { 00ml }\end{array}$ & Absence & Absence & Absence & Absence & Absence & Absence \\
\hline Champignon & Absence & Absence & Absence & $\begin{array}{l}\text { Présence de } \\
\text { champignon }\end{array}$ & $\begin{array}{l}\text { Présence des } \\
\text { champignons }\end{array}$ & Absence \\
\hline $\begin{array}{l}\text { Coliformes } \\
\text { fécaux/100ml }\end{array}$ & Absence & Absence & $\begin{array}{l}\text { Présence des } \\
\text { E. coli }\end{array}$ & Absence & $\begin{array}{l}\text { Présence des } \\
\text { coliformes }\end{array}$ & $<20 \mathrm{ufc} / \mathrm{ml}$ \\
\hline $\begin{array}{l}\text { Moisissures } \\
\text { spores/100ml }\end{array}$ & Absence & Absence & Absence & $\begin{array}{l}\text { Présence de } \\
\text { moisissures et } \\
\text { spores }\end{array}$ & $\begin{array}{l}\text { Présence de } \\
\text { moisissures }+ \\
\text { spores }\end{array}$ & Absence \\
\hline $\begin{array}{l}\text { Pseudomonas } \\
\text { Aérugenosaes } \\
\text { /100ml }\end{array}$ & Absence & Absence & $\begin{array}{l}\text { Présence de } \\
\text { Pseudomonas } \\
\text { Aérugenosae }\end{array}$ & $\begin{array}{l}\text { Présence de } \\
\text { Pseudomonas } \\
\text { Aérugenosae }\end{array}$ & $\begin{array}{l}\text { Présence de } \\
\text { Pseudomonas } \\
\text { Aérugenosae }\end{array}$ & Absence \\
\hline $\begin{array}{l}\text { Enterobacters/ } \\
\text { 100ml }\end{array}$ & Absence & Absence & Absence & Absence & Absence & Absence \\
\hline $\begin{array}{l}\text { Streptocoques } \\
\text { fécaux } / 100 \mathrm{ml}\end{array}$ & Absence & Absence & Absence & Absence & Absence & $<20 \mathrm{ufc} / \mathrm{ml}$ \\
\hline
\end{tabular}

(Culture faite sur milieu Sartorius)

Tableau 4: Evaluation de la vulnérabilité par la méthode DRASTIC des eaux souterraines de la ville de Dschang.

\begin{tabular}{llll}
\hline $\begin{array}{l}\text { Localisation par rapport au } \\
\text { relief }\end{array}$ & $\begin{array}{l}\text { Indice de } \\
\text { vulnérabilité } \\
\text { DRASTIC }\end{array}$ & $\begin{array}{l}\text { Degré de vulnérabilité, } \\
\text { d'après Aller } \text { et al. } \\
(\mathbf{1 9 8 7})\end{array}$ & $\begin{array}{l}\text { Degré de } \\
\text { vulnérabilité, d'après } \\
\text { Hamza } \text { et al. } \mathbf{( 2 0 0 8 )}\end{array}$ \\
\hline $\begin{array}{l}\text { Zone basse sur sol hydromorphes } \\
\text { (bas de versant) }\end{array}$ & 163 & Moyenne & Elevé \\
\hline $\begin{array}{l}\text { Zone basse sur ignimbrite (bas } \\
\text { de versant) }\end{array}$ & 148 & Faible & Elevé \\
\hline $\begin{array}{l}\text { Zone moyenne sur granitogneiss } \\
\text { (sur versant) }\end{array}$ & 115 & Faible & Moyenne \\
\hline $\begin{array}{l}\text { Zone moyenne sur basalte (sur } \\
\text { versant) }\end{array}$ & 146 & Faible & Elevé \\
\hline $\begin{array}{l}\text { Zone haute sur basalte (sommet } \\
\text { du versant) }\end{array}$ & 112 & Faible & Moyenne \\
\hline $\begin{array}{l}\text { Zone haute sur granitogneiss } \\
\text { (sommet du versant) }\end{array}$ & 88 & Faible & Faible \\
\hline Nappe de fracture & 79 & Très faible & Faible \\
\hline
\end{tabular}



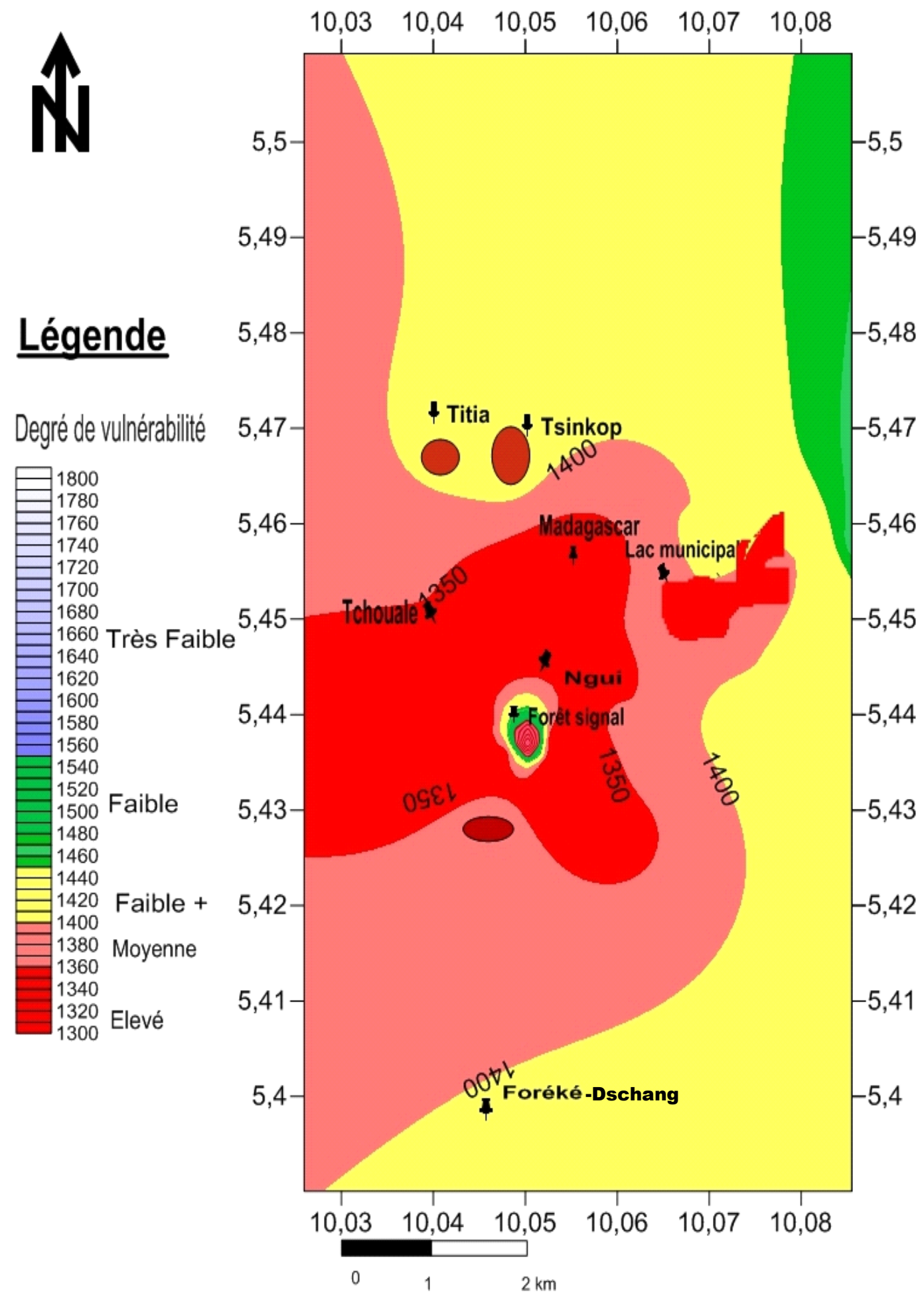

Figure 4: Esquisse de carte de vulnérabilité des nappes souterraines dans la ville de Dschang. 


\section{Conclusion}

La vulnérabilité ne dépend que des seules conditions physiographiques et hydrogéologiques. Un aquifère peut être vulnérable, mais ne pas être atteint par la pollution à l'absence des pressions anthropiques. Afin d'évaluer la vulnérabilité des eaux souterraines à la pollution dans la ville de Dschang, un ensemble de paramètres est pris en compte. Les polluants physiques, chimiques microbiologiques présents dans les eaux souterraines dépendent des grandeurs physiques du sol et des paramètres de la nappe en relation étroite avec le sens de circulation des eaux. La pollution des eaux de surface et des eaux souterraines est essentiellement d'origine anthropique et gouvernée par le sens de circulation des eaux. Les cours d'eaux bien qu'étant des dépotoirs d'ordures et de vidange des WC à canon, demeure les lieux par excellence de lessive et de vaisselle par les populations. Les bas-fonds sont exploités pour la réalisation de l'agriculture urbaine et péri urbaine. Le danger sanitaire est donc grand et permanant. Le sens majeur de circulation est orienté NW - SE, N - S, NE $\mathrm{SW}$ pour se concentrer au $\mathrm{SW}$ où elle circule pour se décharger en partie au niveau de l'escarpement de Foréké - Dschang. Les nappes de la zone d'étude présentent des indices de vulnérabilité variés: faible, moyen et élevé. L'implantation des ouvrages hydrauliques dans les zones hautes de la ville est donc désavantageuse car ce sont des zones préférentielles de protection et de conservation pour favoriser la recharge des nappes. Les sources de pollutions doivent dont être identifiées et éliminées. Il sera judicieux dans l'avenir de préserver les sommets de pentes hydrauliques par une préservation de la végétation ou création de jardins publics au sommet des surfaces topographiques élevées afin de favoriser la recharge des nappes, limiter le ruissellement qui ravine la surface topographique et l'inondation dans les bas-fonds. $\mathrm{La}$ réalisation des constructions dans les basfonds et le long des cours d'eaux sur environ deux mètres de part et d'autre des drains doit être proscrite afin de permettre à la nature de réguler ces eaux et les auto-épurer.

\section{CONFLIT D'INTERETS}

Les auteurs déclarent qu'ils n'ont aucun conflit d'intérêts pour ce manuscrit.

\section{CONTRIBUTIONS DES AUTEURS}

Travail initié et réalisé par FFM en collaboration avec ET. PDK et SNK ont collecté les données de terrain.

\section{REMERCIEMENTS}

Nous remercions l'université de Dschang via le Laboratoire d'Analyse des Sols et de Chimie de l'Environnement (LABASCE) pour avoir analysé les eaux et les sols pour cette étude; la Délégation Départementale du Ministère de l'Eau et de l'Energie de la Menoua a fourni les facilités d'obtention des données de terrain.

\section{REFERENCES}

Akenji VN, Ako Ako A, Akoachere RA II, Takahiro H. 2015. DRASTIC-GIS model for assessing vulnerability to pollution of the phreatic aquiferous formations in Douala-Cameroon. $J$ Afr Earth Sci, 102: 180-190. DOI: https://doi.org/10.1016/j.jafrearsci.11.0 01

Alan W, Rogers P. 2003. Effective water governance, Global Water Partnership, technical committee. TEG background paper, $\mathrm{N}^{\circ} 7,48$ pages.

Aller L, Bennet T, Lehr JH, Petty RTJ, Hackett G. 1987. DRASTIC: a standardized system for evaluating ground water pollution potential using hydrogeology settings. US Environmental Protection Agency, Ada, OK, EPA/600\&2-878-036.

Allier D, Vittecoq B, Mardhel V. 2008. Evaluation de la vulnérabilité intrinsèque des eaux souterraines de la Martinique. Rapport BRGM/RP56283-FR. 81 p.

Baba-Moussa A. 1994. Etude de la pollution bactériologique de la nappe phréatique à partir d'une latrine en Afrique subtropicale. Thèse de doctorat $\left(\mathrm{N}^{\circ} 1276\right)$ es-sciences technologique. Ecole polytechnique fédérale de Lausanne, 256p.

Bézèlgues S, Garets E, Mardhel V, Dörfliger 
N. 2002. Cartographie de la vulnérabilité de Grand-Terre et de Marie-Galatie (Guadeloupe). Phase 1: Méthodologie de détermination de la vulnérabilité, rapport, $45 \mathrm{p}$.

Boufekane A et Saighi O. 2013: Assessment of groundwater pollution by nitrates using intrinsic vulnerability methods: A case study of the Nil valley groundwater (Jijel, North-East Algeria). Afr. J. Environ. Sci. Technol., 7(10): $\quad 949-960 . \quad$ DOI: 10.5897/AJEST2013.1428

Bouyo HM. 2001. Étude pétrographique et structurale des formations de la ville de Dschang et de ses environs (OuestCameroun). Mém. Maît. Univ. Dschang, Cameroun.

Chenini I, Zghibi A, Kouzana L. 2015. Hydrogeological investigations and groundwater vulnerability assessment and mapping for groundwater resource protection and management: state of the art and a case study. $J$ Afr Earth Sci, 109: 11-26. DOI: https://doi.org/10.1016/j.jafrearsci.05.0 08

Djeuda-Tchapnga HB, Tanawa E, Ngnikam E. 2001. L'eau au Cameroun; Tome 1: Approvisionnement en Eau Potable. Press. Univ. : Ydé, Cameroun ; 359p.

Ewodo Mboudou G, Ombolo A, Bon AF, Ntep F, Bineli E. 2016. Apport des méthodes paramétriques DRASTIC, GOD et SI à l'évaluation de la vulnérabilité intrinsèque dans les aquifères du bassin versant de l'Abiergué (région de Yaoundé). Science de la vie, de la terre et agronomie. REV. CAMES, 04(02).

Hamza TH, van Houwelingen HC, Stijnen T. 2008. The binomial distribution of meta-analysis was preferred to model within-study variability. J. Clin. Epidemiol., 61 : 41-51.

Kaliraj S, Chandrasekar N, Simon Peter T, Selvakumar S, Magesh NS. 2014. Mapping of coastal aquifer vulnerable zone in the south west coast of Kanyakumari, South India, using GISbased DRASTIC model. Environ Monit Assess, 187: 4073. DOI: https://doi.org/10.1007/s10661-0144073-2.

Kenfack PL, Tematio P, Kwekam M, Ngueutchoua G, Njike PR. 2011. Evidence of a Miocene volcanosedimentary lithostratigraphic sequence at Ngwa (Dschang Region, West Cameroon): Preliminary analyses and geodynamic interpretation. Journal of Petroleum Technology and Alternative Fuels, 2: 25-34.

Kwekam M. 2005. Genèse et évolution des granitoïdes calco-alcalins au cours de la tectonique panafricaine: le cas des massifs syn à tardi-tectoniques de l'Ouest Cameroun (Régions de Dschang et de Kekem). Thèse Doct. D'Etat, Univ. Yaoundé I, Cameroun. 194p.

Lallemand-Barres. 1994. Normalisation des critères d'établissement des cartes de vulnérabilité aux pollutions. Etude documentaire préliminaire. R37928. BRGM. 18 p.

Loukman B, Nakolendousse S, Nour AM, Memti MN. 2017. Caractérisation hydrochimique de la nappe de Yao et ses environs: relations entre eaux de surface (lac-Fitri) et eaux souterraines. Int. J. Biol. Chem. Sci., 11(3): 13361349.

DOI: https://dx.doi.org/10.4314/ijbcs. v11i3.33

Mfonka Z, Ndam Ngoupayou JR, Kpoumie A, Ndjigui1 P-D, Zammouri M, Ngouh AN, Mouncherou OF, Mfochivé1 OF, Rakotondrabe F. 2019. Hydrodynamic and groundwater vulnerability assessment of the shallow aquifer of the Foumban locality (Bamoun plateau, Western-Cameroon). Arabian Journal of Geosciences, 12:165.

Mouncherou OF, Mfochivé OF, Rakotondrabe F. 2019. Hydrodynamic and groundwater vulnerability assessment of the shallow aquifer of the Foumban locality (Bamoun plateau, Western-Cameroon). Arabian Journal of Geosciences, 12:165.

Moussa RS, Alma MMM, Laouali MS, Natatou I, Habou I. 2018. Caractérisation physico-chimique des eaux des aquifères du Continental 
Intercalaire / Hamadien et du Continentalsiems Terminal de la région de Zinder (Niger). Int. J. Biol. Chem. Sci., 12(5): 2395-2411. DOI: http://ajol.info/index.php/ijbcs

Napolitano P, Fabri AG. 1996. Application of Geographic Information Systems in Hydrology and Water Resources Management. In proceeding of the HydroGis'96 Conference IAHS publ. $\mathrm{N}^{\circ}$ 235, pp. 559-566. Vienna, Australia.

Ngako SB. 2002. Hydrochimie et dynamique comparée des eaux de sources sur socle granito-gneissique et des roches volcaniques dans la région de Dschang (Ouest-Cameroun). Mém. Maît. Fac. Sc. Univ. Dschang, Cameroun.51p.

Njueya AK, Likeng JDH, Nono A. 2012. Hydrodynamique et qualité des eaux souterraines dans le bassin sédimentaire de Douala (Cameroun) : cas des aquifères sur formations Quaternaires et Tertiaire. Int. J. Biol. Chem. Sci., 6(4): 1874-1894. DOI: http://dx.doi.org/10.4314/ijbcs.v6i4.41

Ntangmo Tsafack H, Temgoua E, Njine T. 2012. Physico-Chemical and Bacteriological Quality of the Vegetable Watering Water in the Dschang Town, Cameroon. Journal of Environmental Protection, 3: 949-955

Schnebelen N, Platel JP, Le-Nindre Y, Beaudry D, Hoarau A, Dufour P, Benhammouda S. 2002. Gestion des eaux souterraines en Aquitaine année 5. Opération sectorielle. Protection de la nappe de l'Oligocène en région bordelaise. Rapport BRGM/RP-51178FR.

Tamazang C. 2007. The right to water in Cameroon: legal Framework for sustainable utilisation. Paper prepared for the workshop entitled 'Legal Aspects of Water Sector Reforms' to be organised in Geneva from 20 to 21 April 2007 by the International Environmental Law Research Centre (IELRC) in the context of the Research partnership 2006-2009 on water law sponsored by the Swiss National Science Foundation (SNF)

Tematio P, Kengni L, Bitom D, Hodson ME, Fopoussi JC, Leumbe LO, Mpankam HG, Tsozué D. 2004. Soil and their distribution on Bambouto volcanic mountain, West Cameroon High land, Central Africa. Journal of Africa Earth Sciences, 39: 447-457.

Temgoua E, Ngnikam E, Ndongson B. 2009. Drinking water quality: stakes of control and sanitation in the town of Dschang-Cameroon. Int. J. Biol. Chem. Sci., 3: 441-447. http://ajol.info/index.php/ijbcs

Temgoua E, Ntangmo Tsafack H, Pfeifer HR, Njine T. 2015. Teneurs en éléments majeurs et oligoéléments dans un sol et quelques cultures maraîchères de la ville de Dschang, Cameroun. African Crop Science Journal, 23(1): 35 - 44.

Temgoua E, Santiago H, Pfeifer HR. 2010. Pollution bactériologique de la nappe phréatique et des sols par les latrines proches en zone urbaine pauvre. Cas de la ville de Dschang (Cameroun). International Journal of Advanced Studies and Research in Africa, 1: 228242.

Temgoua E. 2011. Chemical and Bacteriological Analysis of Drinking Water from Alternative Sources in the Dschang Municipality, Cameroon. Journal of Environmental Protection, 2: 620-628.

William PC, Mary AC. 2004. Principles of Environmental Sciences: Inquiry and applications (2nd edn). McGraw-Hill Companies, Inc.: NY 10020; 424 p. 